\title{
Nematic Liquid Crystal Locking Menisci
}

\author{
Milan Svetec ${ }^{1,2,3}$ and Mitja Slavinec ${ }^{1,3}$ \\ ${ }^{1}$ Department of Physics, Faculty of Natural Sciences and Mathematics, University of Maribor, Koroška cesta 160, \\ 2000 Maribor, Slovenia \\ ${ }^{2}$ Regional Development Agency Mura Ltd., Lendavska 5a, 9000 Murska Sobota, Slovenia \\ ${ }^{3}$ Pomurska Akademska Znanstvena Unija (PAZU), Lendavska 5a, 9000 Murska Sobota, Slovenia
}

Correspondence should be addressed to Mitja Slavinec; mitja.slavinec@uni-mb.si

Received 16 February 2013; Accepted 5 March 2013

Academic Editor: Mohindar S. Seehra

Copyright (c) 2013 M. Svetec and M. Slavinec. This is an open access article distributed under the Creative Commons Attribution License, which permits unrestricted use, distribution, and reproduction in any medium, provided the original work is properly cited.

We study meniscus driven locking of point defects of nematic liquid crystals confined within a cylindrical tube with free ends. Curvilinear coordinate system is introduced in order to focus on the phenomena of both (convex and concave) types of menisci. Frank's description in terms of the nematic director field is used. The resulting Euler-Lagrange differential equation is solved numerically. We determine conditions for the defects to be trapped by the meniscus.

\section{Introduction}

The field of liquid crystals, since its discovery in late 19th century, developed into a highly interdisciplinary research field [1]. The most known application of liquid crystals is LC displays. In recent times there are many other technologies and applications ready to be placed on the market, such as organic light emitting diodes, organic field effect transistors, and photovoltaic devices [2-6]. The liquid crystal structures are important also in biology, predominantly for the membranes of living cells, but also for some unusual applications as spider silks, which are spun from a lyotropic nematic liquid crystal precursor [7]. The emergence of this intermediate phase is due to the high concentration of rodlike molecules or aggregates in the watery dope solution. Nematic liquid crystals build intermediate phase combining liquidlike fluidity and solid-like orientational order. These phases are formed by a wide variety of materials comprised of rigid rodlike molecules. The orientational order of liquid crystals (LC) results from the spontaneous alignment of their molecules along a common preferred direction called director and described by a unit vector $\vec{n}(\vec{r})$, where $\vec{r}$ is the position vector. The states $\vec{n}$ and $-\vec{n}$ are equivalent because the LC molecules are nonpolar. Director therefore can be thought of as a headless vector. Because many physical properties (e.g., optical, rheological, and mechanical) can be tailored by adjusting their geometric, external, and interfacial constraints (i.e., shape of the container, molecular orientation imposed by the surface, etc.), the nematic LCs can be very useful in various research fields [8]. As indicated before, the orientational order of LC molecules in principle varies with the position; therefore $\vec{n}(\vec{r})$ is valid only locally. For this reason complex orientational textures are formed [8-12]. Textures often contain defects, which usually correspond to regions (points or lines) where the director field $\vec{n}(\vec{r})$ cannot be uniquely defined $[12,13]$. In order to distinguish between line and point defects one introduces the winding number $M$, also called the Frank index $[14,15]$. It measures the net rotation of the director field on encircling a defect clockwise. Stable point defects are (in topological sense) characterized by integer values of $M$ and line defects with half-integers. The static structure of commonly observed defects is relatively well known. In the director field representation $\vec{n}(\vec{r})$ exhibits singularity at a defect site. Consequently, more complex description of nematic ordering is needed. Conventionally, one uses the tensor order parameter $Q$ in order to avoid singular behavior [8]. At the defect site the local elastic distortions are relatively strong, extending roughly over the size given by the relevant order parameter correlation length $\xi[9,10]$. 
In recent years the focus of the research work was oriented towards the dynamics of defects. The most interesting were the coarsening dynamics of the isotropic-nematic quench [16-20] and the annihilation of the defects in confined geometries [16, 21-29]. Here the interaction between defects and the influence of the confinement on the temporal behavior of defects were studied. All studies regarding defects in cylindrical capillaries were carried out far from the endpoints of the capillary. The interaction of the defect with the meniscus (concave or convex) formed at the end of the capillary was tackled only theoretically by Peroli and Virga in [30]. It is supposed that the defect is attracted towards the center of curvature of the meniscus and therefore eventually trapped in the meniscus if it comes closer than a definite capture distance.

So far the model concerning both concave and convex menisci was not established and no attempts were made to support the findings by numerical methods. This is the focus of our contribution.

The plan of the paper is as follows. In Section 2 we present our model and the system. Free energy functional and geometry of the system are discussed. In Section 3 our results are presented and discussed. In Section 4 we summarize our conclusions.

\section{Modeling}

2.1. Free Energy Functional. We restrict our discussion to rodlike LC molecules and describe nematic structures in terms of the tensor order parameter [31]

$$
Q=S\left(\vec{n} \otimes \vec{n}-\frac{I}{3}\right) .
$$

Here $\vec{n}$ is the nematic director field and $S$ nematic order parameter. In the problem of interest we study the impact of menisci curvature on position of defects deep in the nematic's phase. In this case key features are dominated by Frank elasticity. Consequently, the relevant free energy density can be expressed as $[8,31]$

$$
f=\frac{k}{2}\left[(\nabla \cdot \vec{n})^{2}+(\nabla \times \vec{n})^{2}\right] .
$$

Here we have further simplified the description of the system by using one constant approximation, where $k$ represents Frank's nematic elastic constant [8].

We assume that LC is confined within a cylindrical tube with free ends as described in detail in the following. At the whole boundary of the system homeotropic anchoring is enforced. Therefore, LC molecules are locally oriented along the normal of the confining surface.

2.2. Geometry of the System. Our system contains semiinfinite cylinder bounded on one side by a hemispherical cup. Here cup can be oriented upwards or downwards representing convex and concave meniscus, respectively. We introduce an appropriate orthogonal coordinate system, which would allow us to focus on conditions at menisci.
We start our considerations with the concave menisci. Because of the radial symmetry we can restrict ourselves to only a $1 / 4$ of the cylinder, represented in Figure 1(a). Our orthogonal coordinate system is generated on the basis of the two boundary conditions: $\rho_{\cup}(\xi) \sin \theta_{0}=R$, where $\theta_{0}$ is the function of $\xi$ and $\rho_{\cup}(\xi)$ is the free surface radius of curvature. Subscript indicates the concave nature of the system. And at $\xi \rightarrow 0$ it follows that $\rho_{\cup}(\xi) \rightarrow \infty$. Angle $\theta$ is counted from the main cylinder axis and is limited by $\theta_{0}(\xi)$ where $P\left(\xi, \theta_{0}\right)$ is the point on the cylinder wall at $r=R$. Parameter $\xi$ depends on the height of the particular point $P(\xi, \theta)$ and therefore $0 \leq \xi \leq H$, where $H$ is the height of the menisci at the cylinder axis. From Figure 1(a), we can infer also

$$
\rho_{\cup}(\xi)=\varphi_{\cup}(\xi)-\xi \geq 0
$$

Looking at the convex menisci (Figure 1(b)), the geometry is certainly different in contrast to the concave case, but the equations are very similar. So the counterpart of (3) here can be written as

$$
\rho_{\cap}(\xi)=\xi-\varphi_{\cap}(\xi)
$$

where the subscripts suggest the convex nature of both quantities. When $\xi \rightarrow 0$ from Figure 1(b), we can infer that $\rho_{\cap}(\xi) \rightarrow-\infty$.

In both, convex and concave menisci for $\xi=H$, there is a typical finite radius of curvature $\rho(H)=a$ which depends on the structure (geometrical and chemical) of the liquid crystal molecules and on the boundary conditions of the system.

2.3. Euler Lagrange Equations. We parameterize the nematic director field as

$$
\vec{n}(\theta, \xi)=\cos \psi \vec{e}_{\theta}+\sin \psi \vec{e}_{\xi}
$$

Here $\psi$ is the angle between the local director and the cylindrical axis, and $\vec{e}_{\theta}$ and $\vec{e}_{\xi}$ are unit vectors in the local coordinate system represented in Figure 1(c). In terms of the parameterization introduced, we calculate the free energy density (concave and convex menisci give the same result):

$$
\begin{aligned}
f=\frac{k}{2}\left[\frac{1}{\rho^{2}}\left(1-\frac{\partial \psi}{\partial \theta}\right)^{2}\right. \\
\left.\quad+\frac{\left((1 / \rho) \varphi^{\prime} \sin \theta(1-(\partial \psi / \partial \theta))+(\partial \psi / \partial \xi)\right)^{2}}{\left(1+\varphi^{\prime}(\cos \theta-1)\right)^{2}}\right],
\end{aligned}
$$




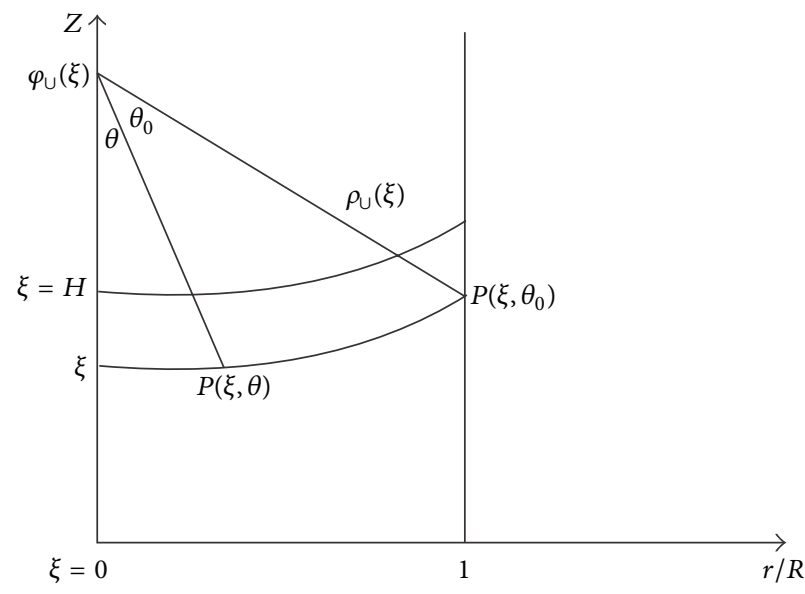

(a)

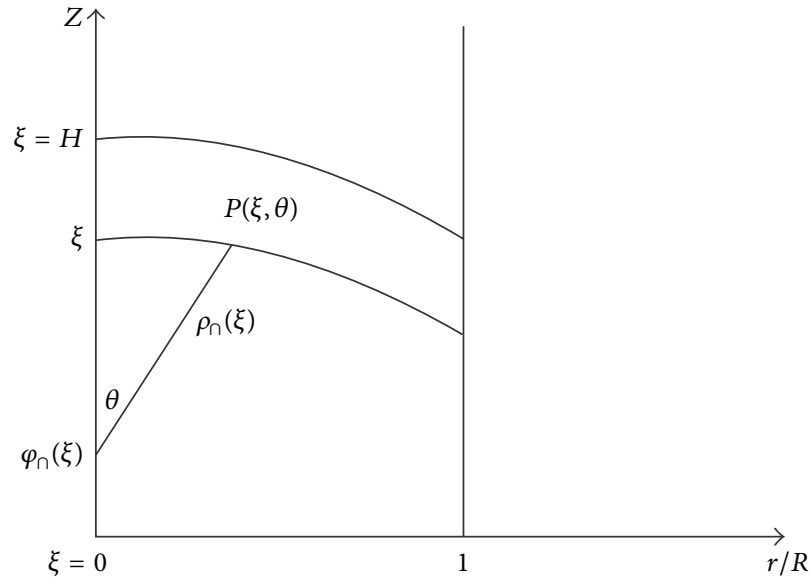

(b)

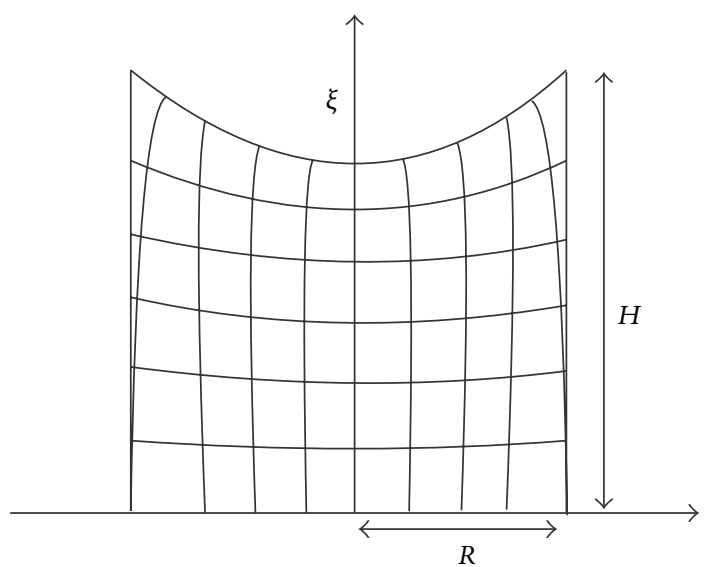

(c)

FIGURE 1: (a) Geometry in the case of the concave meniscus, where the radius of curvature $\rho_{\cup}(\xi)$ is expressed with the local coordinate $\xi$ and the center of meniscus $\varphi_{\cup}(\xi)$. (b) The geometry of the convex case. (c) The orthogonal coordinate system grid for the concave case.

where $\varphi^{\prime}=\partial \varphi / \partial \xi$. The free energy is given by

$$
\begin{aligned}
F=k \int \sin \theta & \\
& \times\left\{\left(1-\frac{\partial \psi}{\partial \theta}\right)^{2}\left(1+\varphi^{\prime}(\cos \theta-1)\right)\right. \\
& \left.+\frac{\left(\varphi^{\prime} \sin \theta(1-(\partial \psi / \partial \theta))+\rho(\partial \psi / \partial \xi)\right)^{2}}{1+\varphi^{\prime}(\cos \theta-1)}\right\} d \theta d \xi .
\end{aligned}
$$

The corresponding Euler-Lagrange equation is given by

$$
\frac{\partial g}{\partial \psi}-\frac{\partial}{\partial \theta} \frac{\partial g}{\partial(\partial \psi / \partial \theta)}-\frac{\partial}{\partial \xi} \frac{\partial g}{\partial(\partial \psi / \partial \xi)}=0
$$

where

$$
\begin{aligned}
& g=\sin \theta\left\{\left(1-\frac{\partial \psi}{\partial \theta}\right)^{2}\left(1+\varphi^{\prime}(\cos \theta-1)\right)\right. \\
&\left.+\frac{\left(\varphi^{\prime} \sin \theta(1-(\partial \psi / \partial \theta))+\rho(\partial \psi / \partial \xi)\right)^{2}}{1+\varphi^{\prime}(\cos \theta-1)}\right\} .
\end{aligned}
$$

Using (8) to minimize (9) we get the Euler-Lagrange differential equation in the following form:

$$
\begin{aligned}
& \cos \theta\left(1-\psi_{\theta}\right)\left(1+\varphi^{\prime}(\cos \theta-1)\right) \\
& -\sin \theta\left(1+\varphi^{\prime}(\cos \theta-1)\right) \psi_{\theta \theta}-\varphi^{\prime}\left(1-\psi_{\theta}\right) \sin ^{2} \theta \\
& +\frac{1}{1+\varphi^{\prime}(\cos \theta-1)} \sin \theta\left(2 \varphi ^ { \prime } \operatorname { c o s } \theta \left(\varphi^{\prime} \sin \theta\left(1-\psi_{\theta}\right)\right.\right. \\
& \left.\left.+(\varphi-\xi) \psi_{\xi}\right)\right)
\end{aligned}
$$




$$
\begin{aligned}
& +\sin \theta \varphi^{\prime}\left(\varphi^{\prime} \cos \theta-\varphi^{\prime} \psi_{\theta} \cos \theta-\varphi^{\prime} \psi_{\theta \theta} \sin \theta\right. \\
& \left. \pm(\varphi-\xi) \psi_{\xi \theta}\right) \\
& \mp\left(\varphi^{\prime}-1\right)\left(\varphi^{\prime} \sin \theta\left(1-\psi_{\theta}\right)+\rho \psi_{\xi}\right) \\
& \mp(\varphi-\xi)\left(\varphi^{\prime \prime} \sin \theta\left(1-\psi_{\theta}\right)-\varphi^{\prime} \psi_{\xi \theta} \sin \theta \pm(\varphi-\xi) \psi_{\xi \xi}\right. \\
& \left.\quad \pm\left(\varphi^{\prime}-1\right) \psi_{\xi}\right) \\
& -\frac{1}{\left(1+\varphi^{\prime}(\cos \theta-1)\right)^{2}} \\
& \times \sin \theta\left(\varphi^{\prime} \sin \theta\left(1-\psi_{\theta}\right) \pm(\varphi-\xi) \psi_{\xi}\right) \\
& \times\left(\varphi^{\prime 2} \sin ^{2} \theta \mp(\varphi-\xi) \varphi^{\prime \prime}(\cos \theta-1)\right)=0 .
\end{aligned}
$$

Here subscripts represent the derivatives according to the appropriate coordinates. The $\varphi^{\prime}$ and $\varphi^{\prime \prime}$ could therefore be written also as $\varphi_{\xi}$ and $\varphi_{\xi \xi}$, respectively. In (10) we already considered both cases ((3) and (4)); therefore the upper signs (for \pm and $\mp$ ) stand for concave menisci and the lower ones for the convex menisci. Appropriate equation, regarding concave and convex cases, was used to numerically test the behavior of the defects enclosed by the cylinder and exposed to the predicted boundary conditions. The results are summarized in the next subsection.

\section{Numerical Results}

To solve (10) we use the Newton-Raphson method. Henceforth we used the following nondimensional quantities: $h=$ $H / R$ (length of the capillary), $a=\rho / R$ (meniscus curvature), and $W=f / f_{\max }$. (free energy density), where $f_{\max }$. is the maximum free energy density of the considered system structure.

In the first part of the work we were focused on the free energy density calculations testing the existence of predicted [30] "capture distance" for the convex case. Calculations were carried out modifying the height $h$ of the cylinder and the free surface radius of curvature $a$. The corresponding free energy density scans showed that the defect stabilizes at the position where there is a minima of the free energy density.

The defect is then locked by the meniscus as depicted in Figure 2. But for this to happen, the length of the capillary $h$ has to be large enough. If $h$ is too small, the free energy density will have its minimal value at the center of the capillary. In Figure 2 there are three different lengths of the capillary represented. The value of the meniscus curvature $a$ equals 2,0 in all three cases. For $h<15$ the defect stabilizes at the center of the capillary. The relative position $p$ represents the $z$ coordinate at the capillary axis. It is calculated as $p=2 z / h-1$, where $z \geq h / 2$. For the center of the capillary it holds $p=0$ and for the edge of the capillary $(z=h)$ it would be $p=1$. From Figure 2 we can infer that the difference in free energy density at the center of the capillary and at the point where the free energy density is minimal increases with the length

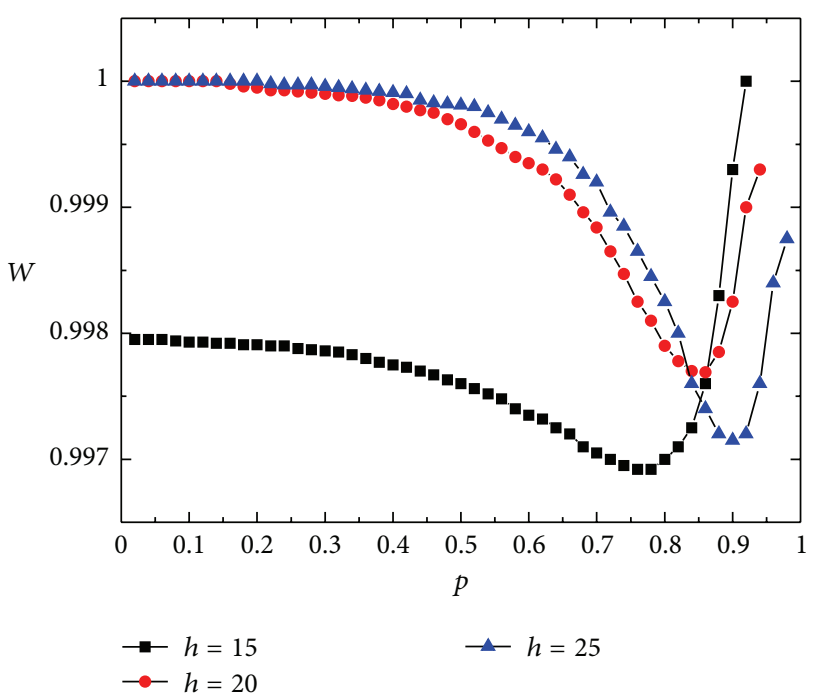

FIGURE 2: The defects stabilize on the cylinder axis at the position where the free energy density is minimal. Here the free energy density $W$ at the relative position $p$ on the axis of the capillary for three different cylinder lengths $h$ is shown. The meniscus curvature $a=2,0$ is held constant.

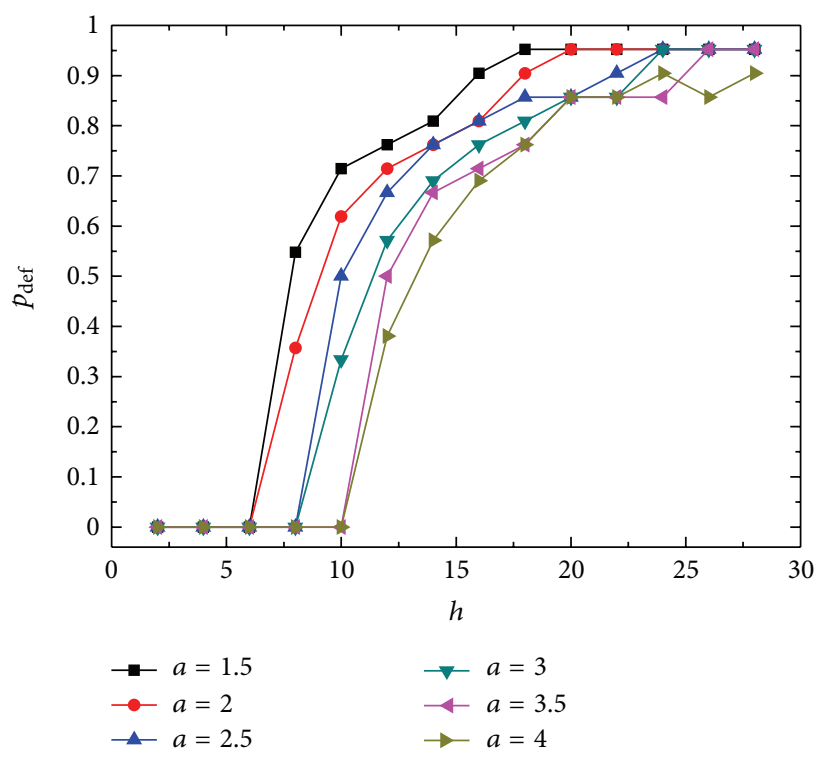

Figure 3: The defects stabilize at the relative position $p_{\text {def }}$ on the cylinder axis, where $p_{\text {def }}=0$ means the center of the capillary and $p_{\text {def }}=1$ the end of it. Here the position of the defect $p_{\text {def }}$ against the length of the capillary $h$ at different meniscus curvatures $a$ is shown.

of the capillary. This means that the defects at increasing $h$ are more and more stabilized. The other conclusion that we can make from Figure 2 is that the position of the free energy density minima at increasing $h$ creeps toward the meniscus meaning that the defect structure is interacting with the meniscus more and more strongly. Now we can ask ourselves if the creeping of the defect towards the meniscus ever ends. The answer we can find in Figure 3. 


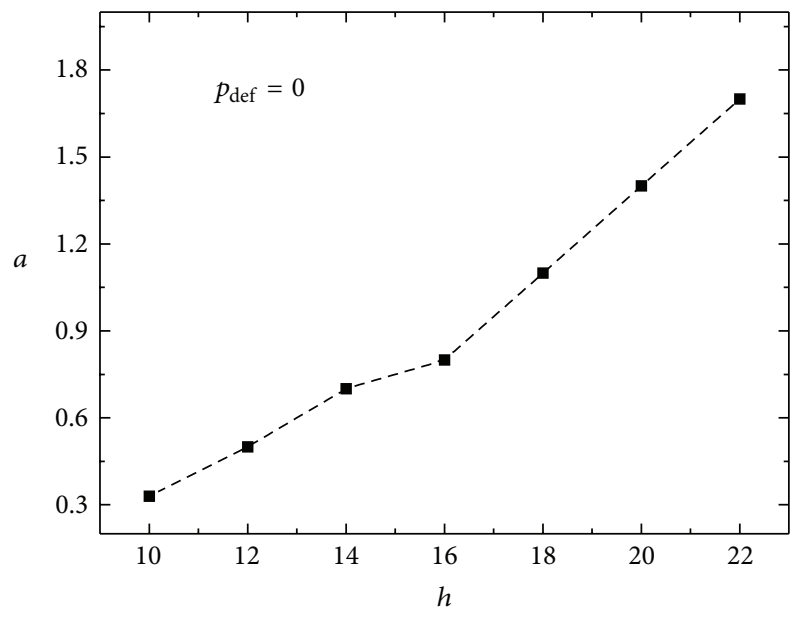

FIGURE 4: In the figure the critical values of meniscus curvature $a$ and the capillary length $h$ are given for which the defect in its equilibrium position is barely attached to the center of the capillary $\left(p_{\text {def }}=0\right)$.

The position of the defect near the meniscus is locked for the capillaries, which exceed certain length, depending on the meniscus curvature $a$. Lower meniscus curvature means higher rate of the defect moving away from the center of the capillary. In Figure 3 we can see also that the systems with lower meniscus curvature reach the final position of the defect at the lower capillary lengths. We can say that the force exerted upon defect is higher if the meniscus curvature is shorter. If the length of the capillary is increased even further, there is no change in the position of the defect regardless of the meniscus curvature. The defect has reached the capture distance [30], and we can say that the defect was trapped in the meniscus. For all capillaries the final $p_{\text {def }}$ is approximately at the 0,95 of its length. That result is also in line with the theoretical prediction of Peroli and Virga [30].

We already could infer from Figure 3 that the meniscus curvature is the "driving force" for the defects to reach their equilibrium positions. Let us examine what is the nature of that relationship. In Figure 4 we can observe at which capillary length and meniscus curvature the defects detach from the central equilibrium position. In Figure 4 the values of meniscus curvature $a$ and capillary length $h$ are given for which the defect still remains at the center of the capillary. If the meniscus curvature (or the length $h$ ) is increased, then the defect moves from its central position towards meniscus.

Therefore the values of meniscus curvature and capillary length in Figure 4 can be called "critical." From Figure 4 we can infer also that the range of the force pulling the defect towards the meniscus is linearly proportional to the meniscus curvature.

\section{Conclusions}

In this paper we presented theoretical and numerical study of locking the defects by meniscus in capillaries filled with nematic liquid crystal molecules having homeotropic anchoring at the capillary walls. The topological defect within the study is characterized by the winding number (i.e., Frank's Index) $M=1$. The position of defect was calculated in the approximation of equal Frank's elastic constants. Different elastic constants might yield quantitatively different results [32]. Addition quantitative changes are also expected if nematic order parameter spatial variations would be taken into account [33]. However, a qualitative feature of symmetry breaking phenomenon, which is the main focus of the paper, is expected to be conserved. For the theoretical considerations and formulation of the free energy we had to describe the system using special curvilinear coordinate system depicted in Figure 1(c). Using Euler-Lagrange differential equation, we numerically calculated equilibrium structures and checked the theoretical predictions, made by Peroli and Virga [30]. In fact the defects are trapped by meniscus as predicted, but certain conditions have to be met. First of all the capillary has to be long enough to avoid the interaction between menisci and secondly the meniscus curvature has to be appropriate to enforce the interaction between meniscus and the defect. The capture distance where defects remain in global equilibrium is very near to the meniscus (Figure 3). On the other hand the range of the force pulling the defect towards the meniscus is proportional to the meniscus curvature (Figure 4).

\section{Acknowledgment}

The authors acknowledge the most fruitful discussions with Professor Virga.

\section{References}

[1] T. J. Sluckin, D. A. Dunmur, and H. Stegemeyer, Crystals That Flow: Classic Papers from the History of Liquid Crystals, Taylor \& Francis, London, UK, 2004.

[2] T. Kato, T. Yasuda, Y. Kamikawa, and M. Yoshio, "Self-assembly of functional columnar liquid crystals," Chemical Communications, no. 7, pp. 729-739, 2009.

[3] S. Laschat, A. Baro, N. Steinke et al., "Discotic liquid crystals: from tailor-made synthesis to plastic electronics," Angewandte Chemie, vol. 46, no. 26, pp. 4832-4887, 2007.

[4] C. Tschierske, "Liquid crystal engineering-new complex mesophase structures and their relations to polymer morphologies, nanoscale patterning and crystal engineering," Chemical Society Reviews, vol. 36, no. 12, pp. 1930-1970, 2007.

[5] S. Sergeyev, W. Pisula, and Y. H. Geerts, "Discotic liquid crystals: a new generation of organic semiconductors," Chemical Society Reviews, vol. 36, no. 12, pp. 1902-1929, 2007.

[6] B. Donnio and D. W. Bruce, Structure and Bonding, Springer, Berlin, Germany, 1999.

[7] E. Atkins, "Biomaterials: silk's secrets," Nature, vol. 424, no. 6952, article 1010, 2003.

[8] P. G. de Gennes and J. Prost, The Physics of Liquid Crystals, Oxford University Press, Oxford, UK, 1993.

[9] S. Kralj, E. G. Virga, and S. Žumer, "Biaxial torus around nematic point defects," Physical Review E, vol. 60, no. 2, Part B, pp. 1858-1866, 1999.

[10] S. Kralj and E. G. Virga, "Universal fine structure of nematic hedgehogs," Journal of Physics A, vol. 34, no. 4, pp. 829-838, 2001. 
[11] P. J. Collings, Liquid Crystals, Nature's Delicate Phase of Matter, Princeton University Press, Princeton, NJ, USA, 2001.

[12] M. Kleman and O. D. Lavrentovich, Soft Matter Physics: An Introduction, Springer, Berlin, Germany, 2002.

[13] P. Oswald and P. Pieranski, Nematic and Cholesteric Liquid Crystals: Concepts and Physical Properties Illustrated by Experiments, CRC Press, Boca Raton, Fla, USA, 2005.

[14] N. D. Mermin, "The topological theory of defects in ordered media," Reviews of Modern Physics, vol. 51, no. 3, pp. 591-648, 1979.

[15] H. R. Trebin, "Defects in liquid crystals and cosmology," Liquid Crystals, vol. 24, no. 1, pp. 127-130, 1998.

[16] A. Pargellis, N. Turok, and B. Yurke, "Monopole-antimonopole annihilation in a nematic liquid crystal," Physical Review Letters, vol. 67, no. 12, pp. 1570-1573, 1991.

[17] I. Chuang, B. Yurke, A. N. Pargellis, and N. Turok, "Coarsening dynamics in uniaxial nematic liquid crystals," Physical Review E, vol. 47, no. 5, pp. 3343-3356, 1993.

[18] J. L. Billeter, A. M. Smondyrev, G. B. Loriot, and R. A. Pelcovits, "Phase-ordering dynamics of the Gay-Berne nematic liquid crystal," Physical Review E, vol. 60, no. 6, Part A, pp. 6831-6840, 1999.

[19] Z. Bradač, S. Kralj, and S. Žumer, "Molecular dynamics study of the isotropic-nematic quench," Physical Review E, vol. 65, 021705, no. 2, Part 1, Article ID 021705, 2002.

[20] Z. Bradač, S. Kralj, and S. Žumer, "Early stage domain coarsening of the isotropic-nematic phase transition," Journal of Chemical Physics, vol. 135, no. 2, Article ID 024506, 2011.

[21] L. M. Pismen and B. Y. Rubinstein, "Motion of interacting point defects in nematics," Physical Review Letters, vol. 69, no. 1, pp. 96-99, 1992.

[22] G. G. Peroli and E. G. Virga, "Annihilation of point defects in nematic liquid crystals," Physical Review E, vol. 54, no. 5, pp. 5235-5241, 1996.

[23] P. E. Cladis and H. R. Brand, "Hedgehog-antihedgehog pair annihilation to a static soliton," Physica A, vol. 326, no. 3-4, pp. 322-332, 2003.

[24] A. Bogi, P. M. Lagarde, I. Dozov, and M. Nobili, "Anchoring screening of defects interaction in a nematic liquid crystal," Physical Review Letters, vol. 89, no. 22, Article ID 225501, 2002.

[25] Z. Bradač, S. Kralj, M. Svetec, and S. Žumer, "Annihilation of nematic point defects: postcollision scenarios," Physical Review E, vol. 67, no. 5. article 050702, Article ID 050702, 4 pages, 2003.

[26] M. Svetec and M. Slavinec, "Structural transition of nematic liquid crystal in cylindrical capillary as a result of the annihilation of two point defects," Journal of Chemical Physics, vol. 128, no. 8, Article ID 084704, 2008.

[27] G. de Luca and A. D. Rey, "Ringlike cores of cylindrically confined nematic point defects," Journal of Chemical Physics, vol. 126, no. 9, Article ID 094907, 2007.

[28] G. DeLuca and A. D. Rey, "Point and ring defects in nematics under capillary confinement," Journal of Chemical Physics, vol. 127, no. 10, Article ID 104902, 2007.

[29] M. Ambrožič, S. Kralj, S. Žumer, T. J. Sluckin, and D. Svenšek, "Annihilation of edge dislocations in smectic-A liquid crystals," Physical Review E, vol. 70, no. 5, Part 1, pp. 51704-51712, 2004.

[30] G. G. Peroli and E. G. Virga, "Modelling the capillary locking of point defects in nematic liquid crystals," IMA Journal of Applied Mathematics, vol. 58, no. 3, pp. 211-236, 1997.

[31] E. G. Virga, Variational Theories for Liquid Crystals, Chapman \& Hall, London, UK, 1994.
[32] S. Žumer and S. Kralj, " $K_{24}$ influence on the structure of the nematic liquid crystal droplets," Liquid Crystal, vol. 12, pp. 613624, 1992.

[33] S. Kralj and S. Žumer, "Saddle-splay elasticity of nematic structures confined to a cylindrical capillary," Physical Review E, vol. 51, no. 1, pp. 366-379, 1995. 

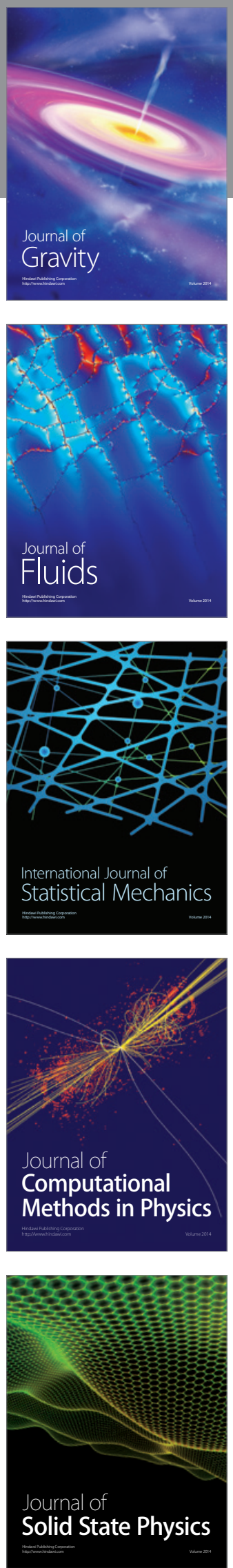

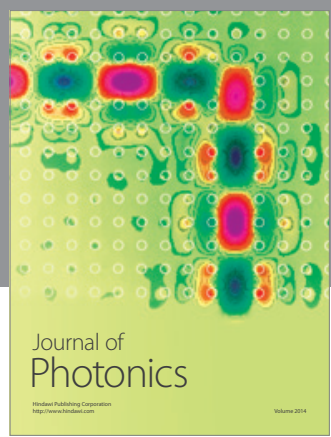

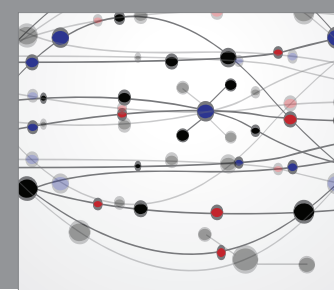

The Scientific World Journal

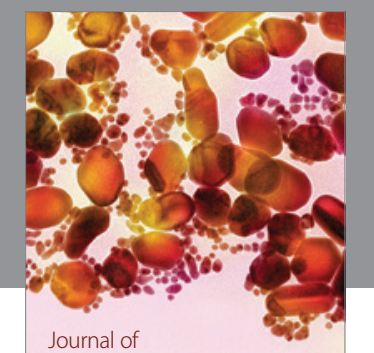

Soft Matter
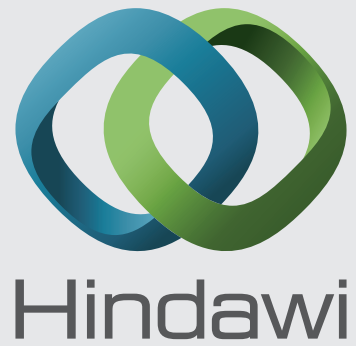

Submit your manuscripts at

http://www.hindawi.com
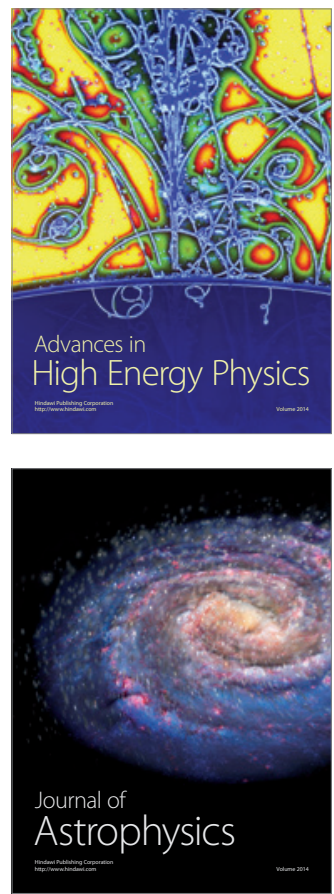
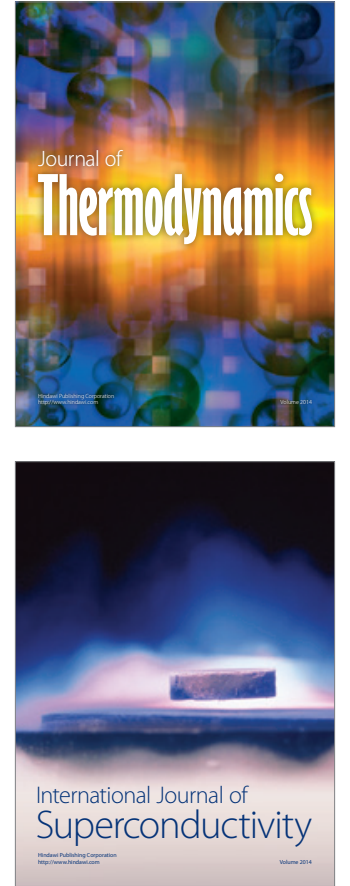
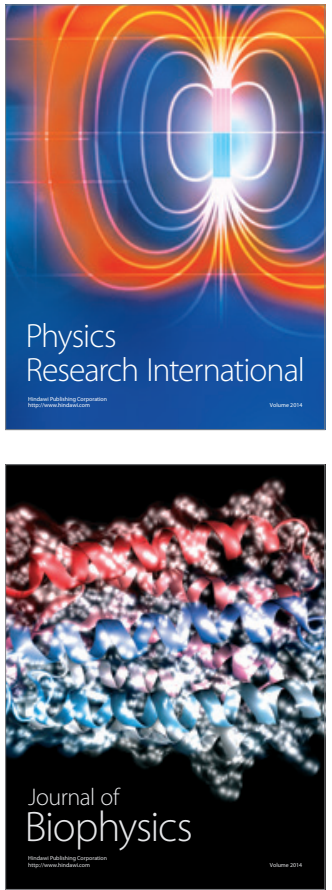
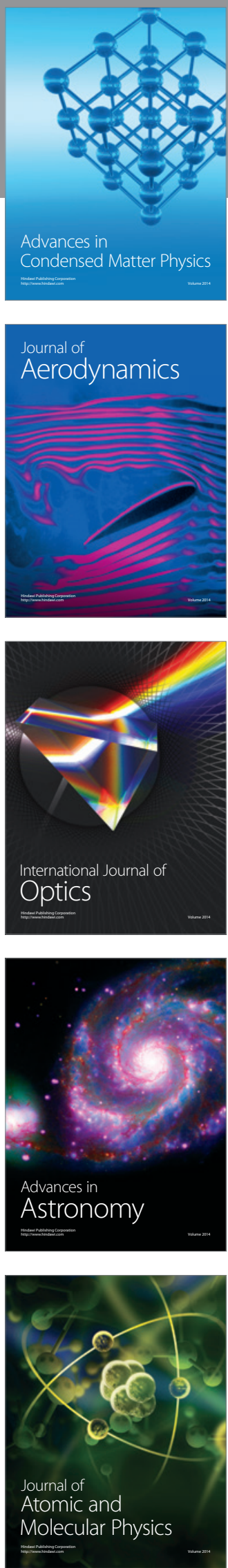